\title{
A Trend-Switching Financial Time Series Model with Level-Duration Dependence
}

\author{
Qingsheng Wang, ${ }^{1,2}$ Aifan Ling, 2 Tao Huang, ${ }^{2}$ \\ Yong Jiang, ${ }^{3}$ and Min Chen ${ }^{4}$ \\ ${ }^{1}$ School of Mathematics and Statistics, Yunnan University, Kunming 650091, China \\ ${ }^{2}$ School of Finance, Jiangxi University of Finance and Economics, Nanchang 330013, China \\ ${ }^{3}$ The Postdoctoral Research Station, Credit Reference Center, The People's Bank of China, \\ Beijing 100031, China \\ ${ }^{4}$ Academy of Mathematics and Systems Science, CAS, Beijing 100190, China
}

Correspondence should be addressed to Qingsheng Wang, ynuwangqingsheng@163.com

Received 25 August 2012; Revised 28 November 2012; Accepted 28 November 2012

Academic Editor: Wei-Chiang Hong

Copyright (C) 2012 Qingsheng Wang et al. This is an open access article distributed under the Creative Commons Attribution License, which permits unrestricted use, distribution, and reproduction in any medium, provided the original work is properly cited.

The financial time series model that can capture the nonlinearity and asymmetry of stochastic process has been paid close attention for a long time. However, it is still open to completely overcome the difficult problem that motivates our researches in this paper. An asymmetric and nonlinear model with the change of local trend depending on local high-low turning point process is first proposed in this paper. As the point process can be decomposed into the two different processes, a high-low level process and an up-down duration process, we then establish the so-called trend-switching model which depends on both level and duration (Trend-LD). The proposed model can predict efficiently the direction and magnitude of the local trend of a time series by incorporating the local high-low turning point information. The numerical results on six indices in world stock markets show that the proposed Trend-LD model is suitable for fitting the market data and able to outperform the traditional random walk model.

\section{Introduction}

The traditional Brownian motion [1] or random walk model [2] has rich applications in physics, biology, and economics, especially in many classical financial theories for capturing the uncertainty of financial data (e.g., efficient market hypothesis). However, the dynamic behavior of Brownian motion is symmetrical and the probability of up and down at any time is always $50 \%$. This is not consistent with the dynamic of price of any risky asset. So Brownian motion is not efficient in capturing the uncertainty for financial time series. To overcome the shortcoming of Brownian motion and capture the uncertainty in financial market, some sophisticated mean-reversion models are proposed, such as, arithmetic Ornstein-Uhlenbeck 
model [3], geometric Ornstein-Uhlenbeck [4], and autoregressive moving average (ARMA) model [5]. In addition, Engle and Granger [6] developed an error correct model (ECM) in which the short-term trend can be corrected by the deviation from the equilibrium of the longterm cointegration. However, Granger and Engle's model has a clear disadvantage: when the process (e.g., prices or returns of financial securities) deviates from a long-term mean level or equilibrium, the direction and magnitude of the local trend will immediately change and it will go back to the long-term mean level or equilibrium. This makes that the change of the trend is too frequent. However, in many time series, such as, sunspot numbers, Canada lynx data, business cycle series, the change of local trend does not occur frequently in a small amplitude or a short period due to the inertia of movement and these time series often present the nonlinearity and asymmetry.

In the last two decades, in order to capture the properties of the real world time series, some regime-switching models, for instance, the threshold autoregressive (TAR) model $[7,8]$, the smoothing transition autoregressive (STAR) model $[9,10]$, and the Markov-switching autoregressive (MSAR) model [11, 12] are paid close attention. The switching between different regimes is often determined by the lagged variables, exogenous variables, or latent variables. For TAR model, the switching from one regime to another depends on the lagged variables or the others in different fixed intervals, such that the threshold values are fixed and the switch of the regimes is abrupt. STAR model is actually a weighted two-regime TAR model, where the weight is a function of the lagged variable or the others such that the transition between regimes is smooth. For MSAR model, The choice of the regime is based on a latent random variable which follows a Markov process. The regime-switching models are more flexible and asymmetric and able to overcome the shortcomings of the mean-reversion models and therefore are used widely in finance (see [13-18]). TAR and STAR models are suitable for the fluctuation data with fixed thresholds or regular cycle, but not for the case that the thresholds and durations of different regimes are time-varying or longer-term dependent, since the change of the regime is still too frequent. While MSAR model characterizes that the choice of regimes is random and only dependent on current state. Hence, in these regimeswitching models, the change of the regime (trend) is always abrupt and does not incorporate the longer-term historical information.

Generally speaking, in financial practice, the measure of the volatility of the returns of risky assets is based on the range of daily price [19-23]. On one hand, for the local high (low) level, technical analysts and investors often exploit the past local high-low levels or resistant-support levels of financial data for predicting the next one. For example, when investors forecast the direction of up-down regime, they often refer to the past local high (low) levels of the technical indicators, for example, BIAS (the relative deviation from the moving average), MACD (moving average convergence divergence), and RSI (relative strength index); on the other hand, for the duration (the arrival time between consecutive events), Engle and Russell [24] proposed an autoregressive conditional duration (ACD) model, and then Bauwens and Giot [25] introduce the logarithmic-ACD model. Zhang et al. [26] extended ACD model to a threshold autoregressive conditional duration (TACD) model. The first two models allow the expected duration to depend linearly on the past ones, but TACD model nonlinearly. Furthermore, Bauwens and Veredas [27] propose the stochastic conditional duration (SCD) model that is more flexible. The applications of the ACD models have been reported in numerous papers (see [28-38]); moreover, for both level and duration, there are numerous empirical applications about cycle (e.g., business cycle, see [39-42] and solar cycle, see [43]), and turning point [44-46]. Durland and McCurdy [47] proposed a class of duration-dependent markov-switching model. The model can incorporate the new 
information (duration) into the transition probabilities of the choice of regimes; however, the new information is not longer term.

In this paper, we introduce a two-regime model that depends on a local high and low turning-point process (local peaks and troughs). This model can be transformed into two processes, a high-low level process and an up-down duration process. The local high (low) level and the up (down) duration of the current regime, which is widely used in many fields, such as economics and finance, depend on those of the past ones. To exploit the past longerterm key information, a new class of trend-switching model that incorporates both a local high-low level process and an up-down duration process is discussed and we call it the trendswitching model which depends on both level and duration (Trend-LD). In fact, both the high-low level process and the up-down duration process are threshold processes. Therefore, Trend-LD model is also called a time-varying double-threshold regime-switching model.

The rest of the paper is organized as follows. In Section 2, we introduce the model specifications. Section 3 gives the estimations of the parameters. In Section 4, we compare the forecasting performances of the proposed model with that of random walk using six stocks indices from different stock markets.

\section{Model Specifications}

In this section, we first provide the definition of local high-low turning point and derive a local high-low level subseries and an up-down duration subseries by which the change in the local trend is determined. And then the equations satisfied by the two subseries are put forward. A nonlinear specifications of the Trend-LD model are proposed.

Let $P_{t}$ denote stock index series at time $t$, and

$$
Y_{t}=\log \left(P_{t}\right)-\mathrm{MA}\left(\log \left(P_{t-1}\right), m\right),
$$

where $\mathrm{MA}\left(\log \left(P_{t-1}\right), m\right)=\sum_{i=t-m}^{t-1} \log \left(P_{i}\right) / m$. Clearly, MA $\left(\log \left(P_{t-1}\right), m\right)$ stands for the $m$ order moving average of the logarithm of index from time $t-m$ to $t-1$, and $Y_{t}$ is the relative bias of the daily stock index at time $t$. In the bias series $Y_{t}$, the long-term trend is removed from the time series of index. As a result, it is relative stable and fluctuate around zero, and it can better represent the short-term relative gains or losses, but not absolute gains or losses, than price $P_{t}$. Figures 1 and 2 give an intuitional example of DJI (US Dow Jones Industrial Average index). In Figure 2, the solid line is the relative bias of the DJI $\left(Y_{t}\right)$; the circles and squares are the local high and low turning-point series, respectively (window radius $w=20$ ).

Definition 2.1. For the relative bias series $\left\{Y_{t}\right\}_{t=1}^{N}, Y_{t_{k}}$ is a local high (low) level, if it is the maxima (minima) of all samples in $\left[t_{k}-w, t_{k}+w\right]$ for a given window radius $w$, $Y_{t_{k}}=\max \left(Y_{t_{k}-w}, \ldots, Y_{t_{k}}, \ldots, Y_{t_{k}+w}\right)\left(\min \left(Y_{t_{k}-w}, \ldots, Y_{t_{k}}, \ldots, Y_{t_{k}+w}\right)\right)$ and we denote the high (low) level $Y_{t_{k}}$ by $H_{k}\left(L_{k}\right)$. The pair $\left(t_{k}, H_{k}\right)\left(\left(t_{k}, L_{k}\right)\right)$ is called a local high (low) turning point.

The local high (low) turning-point process $\left(t_{k}, H_{k}\right)$ can be obtained by scanning all samples of $\left\{Y_{t}\right\}_{t=1}^{N}$. However, it may not be a strictly alternate high-low turning-point series because two neighbor points may be all high (low). In this case, we delete a high (low) point which is not greater (less) than the neighboring point. For simplicity, without loss of generality, suppose the first and last are two local high points, we obtain a strictly alternate local high-low turning-point series $\left\{\left(t_{0}, H_{0}\right),\left(t_{1}, L_{1}\right),\left(t_{2}, H_{1}\right), \ldots,\left(t_{2 n-1}, L_{n}\right),\left(t_{2 n}, H_{n}\right)\right\}$ (see the circles and squares in Figure 2), where the subseries $\left\{t_{0}, t_{1}, \ldots, t_{2 n-1}, t_{2 n}\right\}$ is a series consisting 


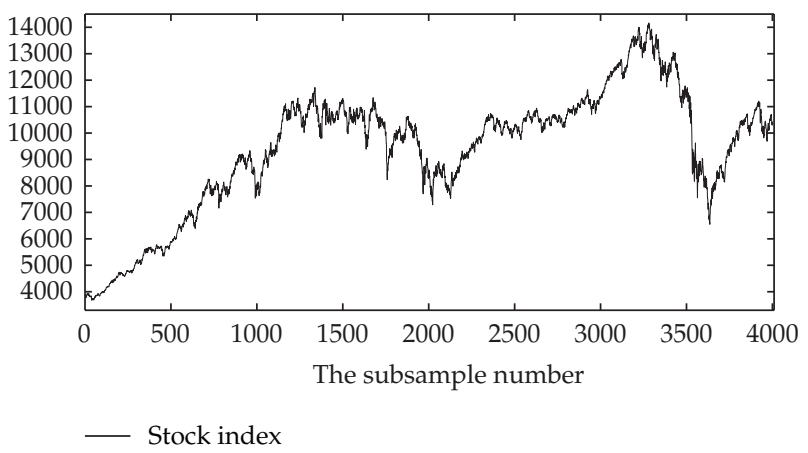

Figure 1: The daily stock index DJI (1994.05.09-2010.08.18).

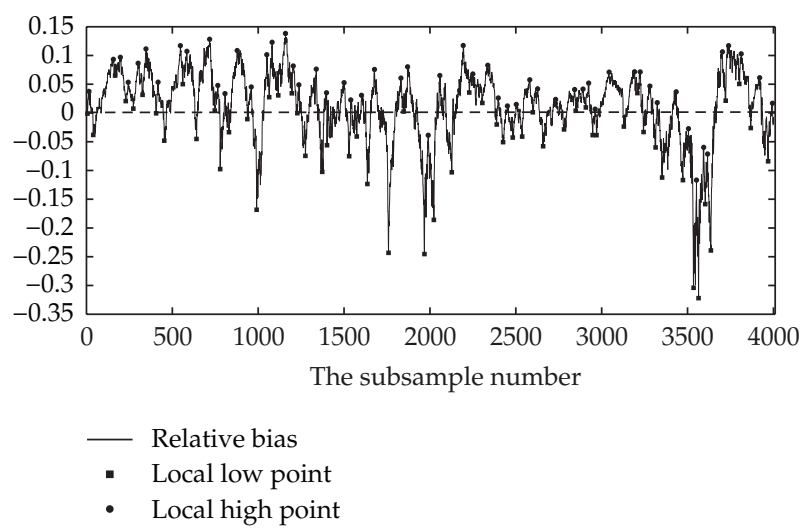

Figure 2: The relative bias of the daily DJI level and its strictly local high-low turning-point series (1994.05.09-2010.08.18).

of time and $\left\{H_{0}, L_{1}, H_{1}, \ldots, L_{n}, H_{n}\right\}$ is another series consisting of the levels of the local high and low points. Furthermore, we can obtain the difference series: let $D_{1}^{-}=t_{1}-t_{0}, D_{1}^{+}=t_{2}-t_{1}$, $D_{2}^{-}=t_{3}-t_{2}, D_{2}^{+}=t_{4}-t_{3}, \ldots, D_{n}^{-}=t_{2 n-1}-t_{2 n-2}, D_{n}^{+}=t_{2 n}-t_{2 n-1}$. We call $D_{i}^{ \pm}$the strictly alternate duration of the up-down trend and $\left\{D_{1}^{-}, D_{1}^{+}, D_{2}^{-}, D_{2}^{+}, \ldots, D_{n}^{-}, D_{n}^{+}\right\}$an up-down duration subseries. Subseries pair $\left\{\left(D_{1}^{-}, L_{1}\right),\left(D_{1}^{+}, H_{1}\right),\left(D_{2}^{-}, L_{2}\right),\left(D_{2}^{+}, H_{2}\right), \ldots,\left(D_{n}^{-}, L_{n}\right),\left(D_{n}^{+}, H_{n}\right)\right\}$ is called Level-Duration (LD) series and will be considered as our main objective.

\subsection{Level-Duration Equations}

Clearly, LD series $\left\{\left(D_{1}^{-}, L_{1}\right),\left(D_{1}^{+}, H_{1}\right),\left(D_{2}^{-}, L_{2}\right),\left(D_{2}^{+}, H_{2}\right), \ldots,\left(D_{n}^{-}, L_{n}\right),\left(D_{n}^{+}, H_{n}\right)\right\}$ controls the direction and magnitude of the local trend of index series $\left\{Y_{t}\right\}$. Because the current local high (low) level and the up (down) duration depend on the past ones. Then, each $H_{k}, L_{k}$ can be expressed as the linear combination of the past high and low level; that is, we have

$$
\begin{gathered}
H_{k}=\pi_{0}^{h}+\sum_{i=1}^{p^{h}} \pi_{1, i}^{h} H_{k-i}+\sum_{j=1}^{q^{h}} \pi_{2, j}^{h} L_{k-j+1}+\eta_{k^{\prime}}^{h} \\
L_{k}=\pi_{0}^{l}+\sum_{i=1}^{p^{l}} \pi_{1, i}^{l} L_{k-i}+\sum_{j=1}^{q^{l}} \pi_{2, j}^{l} H_{k-j}+\eta_{k}^{l} .
\end{gathered}
$$


Similarly, for no nonnegativity constraint on coefficients estimation, we use the logarithm of durations rather than durations to make sure that the range of the logarithm of duration is real $R(-\infty,+\infty)$. the duration subseries $\left\{D_{1}^{-}, D_{1}^{+}, D_{2}^{-}, D_{2}^{+}, \ldots, D_{n}^{-}, D_{n}^{+}\right\}$can be specified by the logarithmic-ACD model [25]

$$
\begin{gathered}
\ln D_{k}^{+}=\pi_{0}^{d^{+}}+\sum_{i=1}^{p^{d^{+}}} \pi_{1, i}^{d^{+}} \ln D_{k-i}^{+}+\sum_{j=1}^{q^{d^{+}}} \pi_{2, j}^{d^{+}} \ln D_{k-j+1}^{-}+\eta_{k}^{d^{+}}, \\
\ln D_{k}^{-}=\pi_{0}^{d^{-}}+\sum_{i=1}^{p^{d^{-}}} \pi_{1, i}^{d^{-}} \ln D_{k-i}^{-}+\sum_{j=1}^{q^{d^{-}}} \pi_{2, j}^{d^{-}} \ln D_{k-j}^{+}+\eta_{k}^{d^{-}},
\end{gathered}
$$

where the error terms $\left\{\eta_{k}^{h}\right\},\left\{\eta_{k}^{l}\right\},\left\{\eta_{k}^{d^{+}}\right\}$, and $\left\{\eta_{k}^{d^{-}}\right\}$are independent normal distributions with mean 0 , variance $\sigma_{h^{\prime}}^{2} \sigma_{l}^{2}, \sigma_{d^{+}}^{2}$, and $\sigma_{d^{-}}^{2}$, respectively. $p^{h}, q^{h}, p^{l}, q^{l}$ and $p^{d^{+}}, q^{d^{+}}, p^{d^{-}}, q^{d^{-}}$are the number of lag order.

Let

$$
\begin{aligned}
\Omega_{k-1}^{h}=\left\{L_{k}, H_{k-1}, L_{k-1}, H_{k-2}, \ldots\right\}, & \Omega_{k-1}^{l}=\left\{H_{k-1}, L_{k-1}, H_{k-2}, L_{k-2}, \ldots\right\}, \\
\Omega_{k-1}^{d^{+}}=\left\{D_{k}^{-}, D_{k-1}^{+}, D_{k-1}^{-}, D_{k-2}^{+}, \ldots\right\}, & \Omega_{k-1}^{d^{-}}=\left\{D_{k-1}^{+}, D_{k-1}^{-}, D_{k-2}^{+}, D_{k-2}^{-}, \ldots\right\},
\end{aligned}
$$

be the information sets. Assume that conditional expectations $h_{k}=E\left(H_{k} \mid \Omega_{k-1}^{h}\right), l_{k}=E\left(L_{k} \mid\right.$ $\left.\Omega_{k-1}^{l}\right), d_{k}^{+}=E\left(D_{k}^{+} \mid \Omega_{k-1}^{d^{+}}\right)$, and $d_{k}^{-}=E\left(D_{k}^{-} \mid \Omega_{k-1}^{d^{-}}\right)$. Then, we have

$$
\begin{gathered}
h_{k}=\pi_{0}^{h}+\sum_{i=1}^{p^{h}} \pi_{1, i}^{h} H_{k-i}+\sum_{j=1}^{q^{h}} \pi_{2, j}^{h} L_{k-j+1}, \\
l_{k}=\pi_{0}^{l}+\sum_{i=1}^{p^{l}} \pi_{1, i}^{l} L_{k-i}+\sum_{j=1}^{q^{l}} \pi_{2, j}^{l} H_{k-j} .
\end{gathered}
$$

By straightforwardly computing, it follows from (2.3) that

$$
\begin{aligned}
& d_{k}^{+}=\exp \left\{\pi_{0}^{d^{+}}+\sum_{i=1}^{p^{d^{+}}} \pi_{1, i}^{d^{+}} \ln D_{k-i}^{+}+\sum_{j=1}^{q^{d^{+}}} \pi_{2, j}^{d^{+}} \ln D_{k-j+1}^{-}+\frac{1}{2} \sigma_{d^{+}}^{2}\right\}, \\
& d_{k}^{-}=\exp \left\{\pi_{0}^{d^{-}}+\sum_{i=1}^{p^{d^{-}}} \pi_{1, i}^{d^{-}} \ln D_{k-i}^{-}+\sum_{j=1}^{q^{d^{-}}} \pi_{2, j}^{d^{-}} \ln D_{k-j}^{+}+\frac{1}{2} \sigma_{d^{-}}^{2}\right\} .
\end{aligned}
$$

In the rest of this paper, we will denote the local high-low level (2.2) by $L\left(p^{h}, q^{h} ; p^{l}, q^{l}\right)$, and the up-down duration (2.3) by $D\left(p^{d^{+}}, q^{d^{+}} ; p^{d^{-}}, q^{d^{-}}\right)$. 


\subsection{Trend-Switching Model with LD Dependence}

In this study, we derive the relative bias $\left\{Y_{t}\right\}$ by removing the middle-to-long term trend (moving-average) from the price. $\left\{Y_{t}\right\}$ is relative stable and fluctuate around 0 , so $\left\{Y_{t}\right\}$ is better at reflecting the short-term relative profit or loss of investors, but not absolute profit or loss, than the price $\left\{P_{t}\right\}$. Furthermore, in finance, researchers and practitioners often pay closer attention to daily return than daily price itself. Similarly, We are interested in the differences of the relative bias rather than the relative bias itself.

Let

$$
r_{t}=Y_{t}-Y_{t-1}
$$

Then, the dynamic characteristics of the relative bias series $\left\{Y_{t}\right\}$ can be indirectly represented by the difference sequences $\left\{r_{t}\right\}$. And assume that the difference sequences $\left\{r_{t}\right\}$ have the following expression:

$$
r_{t}=\left(\mu_{t}^{+}+\varepsilon_{t}^{+}\right) I_{k, t}+\left(\mu_{t}^{-}+\varepsilon_{t}^{-}\right)\left(1-I_{k, t}\right)
$$

where $I_{k, t}$ is an indicator function and satisfies

$$
I_{k, t}= \begin{cases}1, & t \in\left[t_{2 k-1}, t_{2 k}\right), \\ 0, & t \in\left[t_{2 k-2}, t_{2 k-1}\right) .\end{cases}
$$

This means that $I_{k, t}=1$ if $Y_{t}$ is in the $k$ th upward phase, and $I_{k, t}=0$ if $Y_{t}$ is in the $k$ th downward phase. And

$$
\begin{aligned}
& \mu_{t}^{+}=\varphi_{0}^{+}+\sum_{i=1}^{p^{\mu^{+}}} \varphi_{1, i}^{+} r_{t-i}+\varphi_{2}^{+} m_{k, t^{\prime}}^{+} \\
& \mu_{t}^{-}=\varphi_{0}^{-}+\sum_{i=1}^{p^{u^{-}}} \varphi_{1, i}^{-} r_{t-i}+\varphi_{2}^{-} m_{k, t^{\prime}}^{-}
\end{aligned}
$$

where $m_{k, t}^{+}=\left(h_{k}-L_{k}\right) / d_{k}^{+}$and $m_{k, t}^{-}=\left(l_{k}-H_{k-1}\right) / d_{k}^{-}$represent respectively the upward and downward slope in the $k$ th phase; $\varphi_{0}^{ \pm}, \varphi_{1, i}^{ \pm}\left(i=1, \ldots, p^{\mu^{ \pm}}\right)$, and $\varphi_{2}^{ \pm}$are the parameters that need be estimated. The innovation $\left\{\varepsilon_{t}^{+}\right\}$and $\left\{\varepsilon_{t}^{-}\right\}$are mutual independent normal error terms with mean 0 , variance $\sigma_{\varepsilon^{+}}^{2}$ and $\sigma_{\varepsilon^{-}}^{2}$, respectively. We call $(2.8)$ Trend $\left(p^{\mu^{+}} ; p^{\mu^{-}}\right)$.

It is not hard to find that the local trend $\left(\mu_{t}^{+}, \mu_{t}^{-}\right)$consists of three components,

that is, the constant term $\left(\varphi_{0}^{+}, \varphi_{0}^{-}\right)$, the short-term $\left(\sum_{i=1}^{\mu^{\mu^{+}}} \varphi_{1, i}^{+} r_{t-i}, \sum_{i=1}^{p^{\mu^{-}}} \varphi_{1, i}^{-} r_{t-i}\right)$, and the phase term $\left(\varphi_{2}^{+} m_{k, t^{\prime}}^{+}, \varphi_{2}^{-} m_{k, t}^{-}\right)$. The direction and magnitude of the local trend incorporate the key medium- and long-term information, for example, the local high-low levels and the updown durations. Therefore, the dynamic behavior of the equation $\operatorname{Trend}\left(p^{\mu^{+}} ; p^{\mu^{-}}\right)$depends on both the level equation $L\left(p^{h}, q^{h} ; p^{l}, q^{l}\right)$ and the duration equation $D\left(p^{d^{+}}, q^{d^{+}} ; p^{d^{-}}, q^{d^{-}}\right)$. It is integrally called Trend $\left(p^{\mu^{+}} ; p^{\mu^{-}}\right)-L\left(p^{h}, q^{h} ; p^{l}, q^{l}\right)-D\left(p^{d^{+}}, q^{d^{+}} ; p^{d^{-}}, q^{d^{-}}\right)$model, that is, TrendLD model. In fact, the alternate local high-low turning points (the local high-low levels, the 
up-down durations) are the two-dimensional thresholds of the Trend-LD and therefore it is also called the time-varying regime-switching model.

Interestingly, if the parameters of the Trend $\left(p^{\mu^{+}} ; p^{\mu^{-}}\right)$model satisfy some constrain conditions, such as, $\varphi_{0}^{+}=\varphi_{0}^{-}, \varphi_{1, i}^{+}=\varphi_{1, i^{\prime}}^{-}, i=1,2, \ldots, \max \left(p^{\mu^{+}}, p^{\mu^{-}}\right), \varphi_{2}^{+}=\varphi_{2}^{-}=0, \sigma_{\varepsilon^{+}}^{2}=\sigma_{\varepsilon^{-}}^{2}$. Then Trend $\left(p^{\mu^{+}} ; p^{\mu^{-}}\right)$model is a classical pure autoregressive (AR) model. In addition, if the local high-low levels and up-down durations are substituted by some constants, Trend $\left(p^{\mu^{+}} ; p^{\mu^{-}}\right)$ model is a periodically changing AR or a particular form of TAR.

\section{Estimation of Parameters}

In this section, we will estimate the parameters of the Trend-LD model using a two-stage method. We first estimate the parameters of the local high-low level equation $L\left(p^{h}, q^{h} ; p^{l}, q^{l}\right)$ and up-down duration equation $D\left(p^{d^{+}}, q^{d^{+}} ; p^{d^{-}}, q^{d^{-}}\right)$by the method of ordinary least squares (OLS) in the first stage. And then, in the second stage, we estimate the parameters of the equation Trend $\left(p^{\mu^{+}} ; p^{\mu^{-}}\right)$by maximum likelihood (ML). In fact, the ML is equivalent to the OLS under the assumption that error terms are normally distributed. We use the ML in the second stage because the statistical inferences of parameter estimations are similar to that in [48].

The estimation in the first stage is straightforward. Let

$$
\left\{H_{0}, L_{1}, H_{1}, \ldots, L_{n}, H_{n}\right\}, \quad\left\{D_{1}^{-}, D_{1}^{+}, D_{2}^{-}, D_{2}^{+}, \ldots, D_{n}^{-}, D_{n}^{+}\right\}
$$

be the high-low levels and up-down durations series samples data. Then the parameters in equation $L\left(p^{h}, q^{h} ; p^{l}, q^{l}\right)$ and equation $D\left(p^{d^{+}}, q^{d^{+}} ; p^{d^{-}}, q^{d^{-}}\right)$can be estimated directly using OLS method.

After the parameters in equation $L\left(p^{h}, q^{h} ; p^{l}, q^{l}\right)$ are estimated, we can estimate $\left\{\widehat{h}_{k, t}, \widehat{l}_{k, t}\right\}$ using (2.5) and forecast the next local high-low levels. Similarly, in terms of the estimated parameters in equation $D\left(p^{d^{+}}, q^{d^{+}} ; p^{d^{-}}, q^{d^{-}}\right),\left\{\widehat{d}_{k, t^{+}}^{+} \widehat{d}_{k, t}^{-}\right\}$can be obtained using (2.6), and the next local up-down durations can be forecasted. Then, the next local high (low) turning point (threshold point) can be obtained, which is helpful for predicting the change of trend.

In the second stage, we can obtain the estimation of the slope of the up-down phase, $\left\{m_{k, t^{+}}^{+}, m_{k, t}^{-}\right\}$in the equation Trend $\left(p^{\mu^{+}} ; p^{\mu^{-}}\right)$using

$$
m_{k, t}^{+}=\frac{\widehat{h}_{k, t}-L_{k, t}}{\widehat{d}_{k, t}^{+}}, \quad m_{k, t}^{-}=\frac{\widehat{l}_{k, t}-H_{k-1, t}}{\widehat{d}_{k, t}^{-}} .
$$

For the purpose of our analysis, denote $\widehat{M}=\widehat{M}^{-} \cup \widehat{M}^{+}$and $\widehat{M}^{-}=\left\{\widehat{m}_{1}^{-}, \widehat{m}_{2}^{-}, \ldots, \widehat{m}_{n}^{-}\right\}$, $\widehat{M}^{+}=\left\{\widehat{m}_{1}^{+}, \widehat{m}_{2}^{+}, \ldots, \widehat{m}_{n}^{+}\right\}$. Next we estimate the parameters in equation Trend $\left(p^{\mu^{+}} ; p^{\mu^{-}}\right)$. To this end, we denote the parameter set by $\Omega=\Omega^{+} \cup \Omega^{-}$with

$$
\begin{aligned}
& \Omega^{-}=\left\{\varphi_{0}^{-}, \varphi_{1,1}^{-}, \varphi_{1,2^{2}}^{-}, \ldots, \varphi_{1, p^{\mu^{-}}}^{+}, \varphi_{2}^{-}, \sigma_{\varepsilon^{-}}^{2}\right\}, \\
& \Omega^{+}=\left\{\varphi_{0}^{+}, \varphi_{1,1}^{+}, \varphi_{1,2}^{+}, \ldots, \varphi_{1, p^{\mu^{+}}}^{+}, \varphi_{2}^{+}, \sigma_{\varepsilon^{+}}^{2}\right\} .
\end{aligned}
$$


For data set $R_{T_{0}}=\left\{r_{1}, r_{2}, \ldots, r_{T_{0}}\right\}, R_{T_{0}}^{\perp}=\left\{r_{T_{0}+1}, r_{T_{0}+2}, \ldots, r_{T_{2 n}}\right\}$, note that $\varepsilon_{t}^{+}$and $\left\{\varepsilon_{t}^{-}\right\}$are normal series. Then for the given indicator $I_{k, t}$, the conditional logarithm likelihood function can be written as

$$
l\left(R_{T_{0}}^{\perp} \mid R_{T_{0}}, \widehat{M}, \Omega\right)=l\left(R_{T_{0}}^{\perp} \mid R_{T_{0}}, \widehat{M}^{-}, \Omega^{-}\right)+l\left(R_{T_{0}}^{\perp} \mid R_{T_{0}}, \widehat{M}^{+}, \Omega^{+}\right),
$$

where

$$
\begin{aligned}
& l\left(R_{T_{0}}^{\perp} \mid R_{T_{0}}, \widehat{M}^{-}, \Omega^{-}\right) \\
& \quad=-0.5 \sum_{k=1}^{n} \sum_{t=T_{2 k-2}+1}^{T_{2 k-1}}\left[\ln 2 \pi+\ln \sigma_{\varepsilon^{-}}^{2}+\frac{\left(r_{t}-\varphi_{0}^{-}-\sum_{i=1}^{p^{\mu^{-}}} \varphi_{1, i}^{-} r_{t-i}-\varphi_{2}^{-} m_{k, t}^{-}\right)}{\sigma_{\varepsilon^{-}}^{2}}\right], \\
& l\left(R_{T_{0}}^{\perp} \mid R_{T_{0}}, \widehat{M}^{+}, \Omega^{+}\right) \\
& \quad=-0.5 \sum_{k=1}^{n} \sum_{t=T_{2 k-1}+1}^{T_{2 k}}\left[\ln 2 \pi+\ln \sigma_{\varepsilon^{+}}^{2}+\frac{\left(r_{t}-\varphi_{0}^{+}-\sum_{i=1}^{p^{\mu^{+}}} \varphi_{1, i}^{+} r_{t-i}-\varphi_{2}^{+} m_{k, t}^{+}\right)}{\sigma_{\varepsilon^{+}}^{2}}\right] .
\end{aligned}
$$

To obtain the maximum likelihood estimation (MLE) of the conditional logarithm likelihood function $l\left(R_{T_{0}}^{\perp} \mid R_{T_{0}}, \widehat{M}, \Omega\right)$ on parameter set $\Omega$, we only need to maximize $l\left(R_{T_{0}}^{\perp} \mid R_{T_{0}}, \widehat{M}^{-}, \Omega^{-}\right)$ and $l\left(R_{T_{0}}^{\perp} \mid R_{T_{0}}, \widehat{M}^{+}, \Omega^{+}\right)$on parameter set $\Omega^{-}$and $\Omega^{+}$, respectively. If the process $r_{t}$ is stationary, the estimates fall within the boundaries of the allowable parameter space, then the estimators are consistent [48].

\section{Hypotheses of Asymmetry and Nonlinearity}

In this section, we focus on the asymmetry and nonlinearity of Trend-LD model and consider the following three hypotheses.

Hypothesis 1. The dynamic behavior of the high-low level process does not have asymmetric effects on space (level), namely

$$
H_{0}^{1}:\left\{\begin{array}{l}
\pi_{0}^{h}=\pi_{0}^{l}=\pi_{0}^{h l}, \\
\pi_{1, i}^{h}=\pi_{1, i}^{l}=\pi_{1, i^{\prime}}^{h l} \quad i=1,2, \ldots, \max \left\{p^{h}, p^{l}\right\}, \\
\pi_{2, j}^{h}=\pi_{2, j}^{l}=\pi_{2, j^{\prime}}^{h l} \quad j=1,2, \ldots, \max \left\{q^{h}, q^{l}\right\} \\
\sigma_{h}^{2}=\sigma_{l}^{2}=\sigma_{h l}^{2} .
\end{array}\right.
$$


Hypothesis 2. The dynamic behavior of the up-down duration process does not have asymmetric effect on time (duration), namely

$$
H_{0}^{2}:\left\{\begin{array}{l}
\pi_{0}^{d^{+}}=\pi_{0}^{d^{-}}=\pi_{0}^{d}, \\
\pi_{1, i}^{d^{+}}=\pi_{1, i}^{d^{-}}=\pi_{1, i}^{d}, \quad i=1,2, \ldots, \max \left\{p^{d^{+}}, p^{d^{-}}\right\}, \\
\pi_{2, j}^{d^{+}}=\pi_{2, j}^{d^{-}}=\pi_{2, j^{\prime}}^{d} \quad j=1,2, \ldots, \max \left\{q^{d^{+}}, q^{d^{-}}\right\}, \\
\sigma_{d^{+}}^{2}=\sigma_{d^{-}}^{2}=\sigma_{d}^{2} .
\end{array}\right.
$$

Hypothesis 3. The dynamic of the local trend is linear and does not depend on local high-low turning point (level and duration), namely

$$
H_{0}^{3}:\left\{\begin{array}{l}
\varphi_{0}^{+}=\varphi_{0}^{-}=\varphi_{0}, \\
\varphi_{1, i}^{+}=\varphi_{1, i}^{-}=\varphi_{1, i}, \quad i=1,2, \ldots, \max \left\{p^{\mu^{+}}, p^{\mu^{-}}\right\} \\
\varphi_{2}^{+}=\varphi_{2}^{-}=\varphi_{2}=0 \\
\sigma_{\varepsilon^{+}}^{2}=\sigma_{\varepsilon^{-}}^{2}=\sigma_{\varepsilon}^{2}
\end{array}\right.
$$

If Hypothesis 1 is true, the local high-low level equation $L\left(p^{h}, q^{h} ; p^{l}, q^{l}\right)$ can be further simplified to the equation $L(p, q)$. The truth of Hypothesis 2 will show that the up-down duration equation $D\left(p^{d^{+}}, q^{d^{+}} ; p^{d^{-}}, q^{d^{-}}\right)$can be further simplified to the equation $D(p, q)$. Hypothesis 3 means that equation Trend $\left(p^{\mu^{+}} ; p^{\mu^{-}}\right)$can be further simplified to a linear autoregressive model $\operatorname{AR}(p)$. The three Hypotheses are tested by the likelihood ratio statistic which is asymptotic Chi-square distribution and its degree of freedom is the number of the constrained parameters.

\section{Empirical Applications}

Six stock indices with different degree of market development are used to test the performance of our models in this section. These indices are US Dow Jones Industrial Average (DJI), US Standard \& Poor 500 Index (SP500), British Financial Times Stock Exchange 100 Index (FTSE), Singapore Straits Times Index (STI), Indian Bombay Stock Exchange Sensex Index (BSE), and Chinese Shenzhen Component Index (SZCI).

\subsection{Data}

We collect the daily close prices of the above six indices from "http://www.finance.yahoo .com." Each complete data sample is divided into two subsamples, estimating subsample (ES) and forecasting subsample (PS). The details about the data can be found in Table 1. It is worth mentioning here that we preliminarily use $80 \%$ of training set for each index and then select a local high point as the separating point which is nearest from the point that divided the total daily index by $80 \%-20 \%$ since the end of the local high-low turning-point series $\left\{\left(t_{0}, H_{0}\right),\left(t_{1}, L_{1}\right),\left(t_{2}, H_{1}\right), \ldots,\left(t_{2 n-1}, L_{n}\right),\left(t_{2 n}, H_{n}\right)\right\}$, is a local high point.

According to the definition of local high-low turning point, we computed its level data $H_{k}$ and $L_{k}$, duration data $D_{k}^{+}$and $D_{k}^{-}$for each index and provide their descriptive 
Table 1: The periods of estimating subsample (ES) and predicting subsample (PS).

\begin{tabular}{lcccc}
\hline Index & ES & Sizes & PS & Sizes \\
\hline DJI & $1928.10 .01-1993.08 .26$ & 16286 & $1993.08 .27-2010.08 .18$ & 4276 \\
SP500 & $1950.01 .03-1997.12 .04$ & 12062 & $1997.12 .05-2010.08 .18$ & 3193 \\
FTSE & $1984.04 .02-2005.07 .07$ & 5372 & $2005.07 .08-2010.08 .18$ & 1293 \\
STI & $1987.12 .28-2005.10 .12$ & 4445 & $2005.10 .13-2010.08 .18$ & 1215 \\
BSE & $1990.01 .01-2006.10 .17$ & 3932 & $2006.10 .18-2010.08 .18$ & 943 \\
SZCI & $1991.04 .03-2006.07 .04$ & 3757 & $2006.07 .05-2010.08 .18$ & 1008 \\
\hline
\end{tabular}

Table 2: Descriptive statistics of local high-low level and up-down duration.

\begin{tabular}{lccccccc}
\hline & statistic & DJI & SP500 & FTSE & STI & BSE & SZCI \\
\hline high & mean & 0.041 & 0.036 & 0.041 & 0.049 & 0.078 & 0.097 \\
level & std & 0.027 & 0.017 & 0.02 & 0.035 & 0.05 & 0.091 \\
\hline low & mean & -0.046 & -0.037 & -0.042 & -0.055 & -0.074 & -0.091 \\
level & std & 0.046 & 0.035 & 0.04 & 0.055 & 0.056 & 0.069 \\
\hline & level range & 0.087 & 0.073 & 0.083 & 0.104 & 0.152 & 0.188 \\
\hline up & mean & 24.489 & 23.427 & 23.349 & 23.464 & 25.653 & 23.685 \\
duration & std & 13.438 & 12.452 & 12.934 & 12.225 & 13.637 & 12.466 \\
\hline up & mean & 27.817 & 27.073 & 28.287 & 27.071 & 25.221 & 27.88 \\
duration & std & 14.511 & 15.079 & 15.762 & 16.309 & 13.017 & 13.871 \\
\hline & duration cycle & 52.306 & 50.5 & 51.636 & 50.535 & 50.874 & 51.565 \\
\hline
\end{tabular}

Note: level range $=$ mean of local high level - mean of local low level, duration cycle $=$ mean of up duration + mean of down duration.

statistics (mean and standard deviation) in Table 2, where moving average order $m=20$, window radius $w=20$ (The choice of these two parameters is related to the commonly used parameters of Dow's trend theory and Elliott's wave theory in technical analysis. This study is to model the short-term trend and fluctuation of the stock index and to compare with the random walk hypothesis. Therefore, we choose some short-term parameters). In terms of the high-low level, the vibration amplitudes of the later three indices (STI, BSE, SZCI) are greater than those of the first three indices (DJI, SP500, FTSE). Among 6 indices, the fluctuation of the SP500 is the smallest and that of the SZCI is the largest. However, it seems that the characteristics of the up-down durations are not significantly different. Therefore, the local high-low level maybe have more informative than the up-down duration.

\subsection{Estimates and Tests}

For the convenience of comparisons, we preliminarily consider the simple case of the proposed models, Trend $(1 ; 1)-L(2,2 ; 2,2) D(1,1 ; 1,1)$, which are enough to capture the dynamics of the data (in financial application, first- or second-order AR model is normally used, especially in modeling stock returns. For our Trend-LD, we use second-order lag for the level equation, first-order lag for the duration equation, and the autoregressive term of the local trend equation. We did not provide concise explanation how we choose the orders. In general, the choice of the order is based on AIC or BIC criteria. We believe that these criteria are limited as the penalty on the number of parameters is somewhat arbitrary. We estimated the results on different combinations of parameters in unreported tests. For the local trend equation, we found the coefficients were not significantly different from 0 by $t$-test 
Table 3: OLE of high-low level equation.

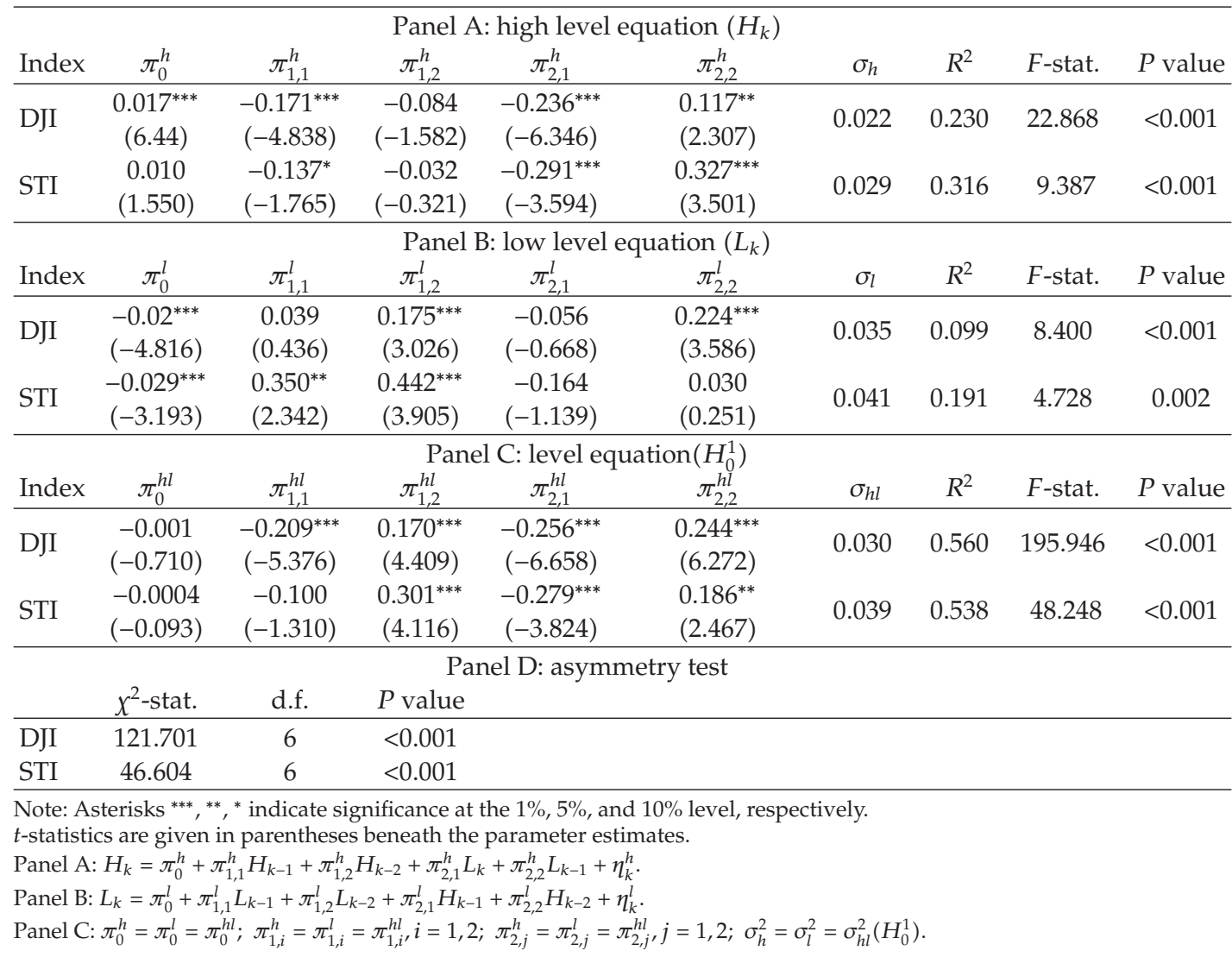

when the order of autoregressive term was 2 or higher, and the conditional expectation of the difference $r_{t}$ is mainly determined by the second term related to turning points. Therefore, we only use the first order of the autoregressive term as well as the choice of lag order of other parameters). Then we estimate the parameters and conduct the asymmetry and nonlinearity tests for the proposed models. Ultimately, the structure of the model is chosen as Trend $(1 ; 1)-L(2,2 ; 2,2) D(1,1)$ after the tests. For brevity, we consider two representative indexes, the developed market index DJI and developing market index STI and focus our discussion on results pertaining to the two indexes. The other four stock indexes have some similar properties.

We first consider the OLS estimations (OLE) and asymmetric tests on high-low level equation $L(2,2 ; 2,2)$ and up-down duration equation $D(1,1 ; 1,1)$. The results are presented in Tables 3 and 4 , respectively. We find (1) for equation $L(2,2 ; 2,2)$ under the null hypothesis $H_{0}^{1}$ (in Panel C) and alternative hypothesis (in Panel A and B), the $R^{2}$ statistics are very large for $L(2,2 ; 2,2)$, and its $P$ values of $F$-statistics are very small. From the $t$ statistics of the coefficients, the current high-low level is significantly dependent on the past (lagged first-order and second-order) high-low levels. (see Table 3). However, for duration equation $D(1,1 ; 1,1)$ under the null hypothesis $H_{0}^{1}$ (in Panel C) and alternative hypothesis (in Panel A and $\mathrm{B}$ ), The $R^{2}$ statistics are very small for duration equation, and its $P$ values of $F$-statistcs are comparatively large (see Table 4 ). From the $t$ statistics of the coefficients, the current updown duration is weakly dependent on the past (lagged first-order) up-down durations. 
Table 4: OLE of up-down duration equation.

\begin{tabular}{|c|c|c|c|c|c|c|c|}
\hline \multicolumn{8}{|c|}{ Panel A: Up duration equation $\left(D_{k}^{+}\right)$} \\
\hline Index & $\pi_{0}^{d^{+}}$ & $\pi_{1,1}^{d^{+}}$ & $\pi_{2,1}^{d^{+}}$ & $\sigma_{d^{+}}$ & $R^{2}$ & F-stat. & $P$ value \\
\hline DJI & $\begin{array}{l}3.029^{* * *} \\
(12.058)\end{array}$ & $\begin{array}{c}0.011 \\
(0.203)\end{array}$ & $\begin{array}{c}0.002 \\
(0.034)\end{array}$ & 0.511 & 0.0001 & 0.021 & 0.979 \\
\hline STI & $\begin{array}{c}3.080^{* * *} \\
(6.466)\end{array}$ & $\begin{array}{c}-0.021 \\
(-0.220)\end{array}$ & $\begin{array}{l}0.0007 \\
(0.006)\end{array}$ & 0.514 & 0.0001 & 0.025 & 0.975 \\
\hline \multicolumn{8}{|c|}{ Panel B: Down duration equation $\left(D_{k}^{-}\right)$} \\
\hline Index & $\pi_{0}^{d^{-}}$ & $\pi_{1,1}^{d^{-}}$ & $\pi_{2,1}^{d^{-}}$ & $\sigma_{d^{-}}$ & $R^{2}$ & F-stat. & $P$ value \\
\hline DJI & $\begin{array}{l}2.824^{* * *} \\
(16.194)\end{array}$ & $\begin{array}{c}-0.023 \\
(-0.562)\end{array}$ & $\begin{array}{l}0.085^{* *} \\
(2.107)\end{array}$ & 0.536 & 0.023 & 3.620 & 0.028 \\
\hline STI & $\begin{array}{c}3.988^{* * *} \\
(7.739)\end{array}$ & $\begin{array}{c}-0.158 \\
(-1.241)\end{array}$ & $\begin{array}{l}-0.130 \\
(-1.201)\end{array}$ & 0.593 & 0.035 & 1.485 & 0.233 \\
\hline \multicolumn{8}{|c|}{ Panel C: Duration equation $\left(H_{0}^{2}\right)$} \\
\hline Index & $\pi_{0}^{d}$ & $\pi_{1,1}^{d}$ & $\pi_{2,1}^{d}$ & $\sigma_{d}$ & $R^{2}$ & $F$-stat. & $P$ value \\
\hline DJI & $\begin{array}{l}2.924^{* * *} \\
(16.194)\end{array}$ & $\begin{array}{c}-0.023 \\
(-0.562)\end{array}$ & $\begin{array}{l}0.085^{* *} \\
(2.107)\end{array}$ & 0.116 & 0.008 & 2.413 & 0.090 \\
\hline STI & $\begin{array}{l}3.555^{* * *} \\
(10.240)\end{array}$ & $\begin{array}{c}-0.090 \\
(-1.181)\end{array}$ & $\begin{array}{c}-0.071 \\
(-0.924)\end{array}$ & 0.553 & 0.012 & 1.041 & 0.355 \\
\hline \multicolumn{8}{|c|}{$\begin{array}{l}\text { Panel D: Asymmetry test } \\
P \text { value }\end{array}$} \\
\hline DJI & 8.967 & 4 & 0.062 & & & & \\
\hline STI & 4.340 & 4 & 0.362 & & & & \\
\hline
\end{tabular}

Note: Asterisks ${ }^{* * *},{ }^{* *},{ }^{*}$ indicate significance at the $1 \%, 5 \%, 10 \%$ level, respectively.

$t$-statistics are given in parentheses beneath the parameter estimates.

Panel A: $\ln D_{k}^{+}=\pi_{0}^{d^{+}}+\pi_{1,1}^{d^{+}} \ln D_{k-1}^{+}+\pi_{2,1}^{d^{+}} \ln D_{k}^{-}+\eta_{k}^{d^{+}}$.

Panel B: $\ln D_{k}^{-}=\pi_{0}^{d^{-}}+\pi_{1,1}^{d^{-}} \ln D_{k-1}^{-}+\pi_{2,1}^{d^{-}} \ln D_{k}^{+}+\eta_{k}^{d^{-}}$.

Panel C: $\pi_{0}^{d+}=\pi_{0}^{d-}=\pi_{0}^{d} ; \pi_{1,1}^{d+}=\pi_{1,1}^{d-}=\pi_{1,1}^{d} ; \pi_{2,1}^{d+}=\pi_{2,1}^{d-}=\pi_{2,1}^{d} ; \sigma_{d+}^{2}=\sigma_{d-}^{2}=\sigma_{d}^{2}\left(H_{0}^{2}\right)$.

The constant estimations $\left(\pi_{0}^{d^{+}}, \pi_{0}^{d^{-}}\right)$are all significantly greater than zero. That means there is inertia in the movement of the stock indices after each high (low) turning point. The findings show that the dependency of the current up-down duration on the past ones is much weaker than that of the current local high-low level on the past ones. The reason may be that the profit of investors is more directly related to local high-low level of the relative bias of stock price than to the up-down duration. (2) The nonsymmetry test in Panel D shows as follows: at the $5 \%$ significance level, the null hypothesis $H_{0}^{1}$ should be rejected, namely, the level equation is not symmetric $L(2,2)$, but asymmetric $L(2,2 ; 2,2)$, it means that there is asymmetric effect on high-low level process (space); however, the null hypothesis $H_{0}^{2}$ fails to be rejected, namely, the duration equation is not asymmetric $D(1,1 ; 1,1)$, but symmetric $D(1,1)$, it means that there is symmetric effect on up-down duration process (time).

Next, we consider the maximum likelihood estimate (MLE) and nonlinearity test on equation Trend $(1 ; 1)$. The results are presented in Table 5 . From the $t$ statistics and the $P$ value of the $F$-statistics in Panels A and B, the coefficient estimations $\widehat{\varphi}_{2}$ of the up-down phase slopes $\left(m_{k}^{+}, m_{k}^{-}\right)$are significant greater than zero under $1 \%$ significant level and are the greatest among of $\hat{\varphi}_{0}, \widehat{\varphi}_{1}, \widehat{\varphi}_{2}$. This means that the local up-down trend is mainly affected by the up-down phase trend $\left(m_{k}^{+}, m_{k}^{-}\right)$. By the non-symmetry test in Panel $\mathrm{D}$, at the $1 \%$ significance level, the null hypothesis $H_{0}^{3}$ is rejected. It shows the dynamic of the local trend 
Table 5: MLE of trend equation.

\begin{tabular}{|c|c|c|c|c|c|c|c|}
\hline \multicolumn{8}{|c|}{ Panel A: up trend equation $\left(r_{t}^{+}\right)$} \\
\hline Index & $\varphi_{0}^{+}$ & $\varphi_{1}^{+}$ & $\varphi_{2}^{+}$ & $\sigma_{\mu^{+}}$ & $R^{2}$ & F-stat. & $P$ value \\
\hline DJI & $\begin{array}{c}0.0007^{* * *} \\
(2.956)\end{array}$ & $\begin{array}{l}-0.0080 \\
(-0.674)\end{array}$ & $\begin{array}{l}0.671^{* * *} \\
(11.606)\end{array}$ & 0.011 & 0.018 & 67.954 & $<0.001$ \\
\hline STI & $\begin{array}{c}0.0004 \\
(0.097)\end{array}$ & $\begin{array}{c}0.013 \\
(0.574)\end{array}$ & $\begin{array}{c}0.893^{* * *} \\
(8.840)\end{array}$ & 0.012 & 0.040 & 42.387 & $<0.001$ \\
\hline \multicolumn{8}{|c|}{ Panel B: down trend equation $\left(r_{t}^{-}\right)$} \\
\hline Index & $\varphi_{0}^{-}$ & $\varphi_{1}^{-}$ & $\varphi_{2}^{-}$ & $\sigma_{\mu^{-}}$ & $R^{2}$ & $F$-stat. & $P$ value \\
\hline DJI & $\begin{array}{c}-0.001^{* * *} \\
(-3.926)\end{array}$ & $\begin{array}{c}-0.113^{* * *} \\
(-9.761)\end{array}$ & $\begin{array}{c}0.538^{* * *} \\
(6.605)\end{array}$ & 0.012 & 0.0150 & 64.694 & $<0.001$ \\
\hline STI & $\begin{array}{c}-0.002^{* * *} \\
(-3.759)\end{array}$ & $\begin{array}{c}0.008 \\
(0.389)\end{array}$ & $\begin{array}{c}0.227^{*} \\
(1.925)\end{array}$ & 0.013 & 0.0017 & 1.965 & 0.140 \\
\hline \multicolumn{8}{|c|}{ Panel C: trend equation $\left(H_{0}^{3}\right)$} \\
\hline Index & $\varphi_{0}$ & $\varphi_{1}$ & & $\sigma_{\mu}$ & $R^{2}$ & $F$-stat. & $P$ value \\
\hline DJI & $\begin{array}{c}-1.74 E-6 \\
(-0.019)\end{array}$ & $\begin{array}{c}0.0265^{* * *} \\
(3.367)\end{array}$ & & 0.012 & 0.001 & 11.334 & $<0.001$ \\
\hline STI & $\begin{array}{c}-1.31 E-5 \\
(-0.067)\end{array}$ & $\begin{array}{c}0.124^{* * *} \\
(8.218)\end{array}$ & & 0.013 & 0.015 & 67.537 & $<0.001$ \\
\hline \multicolumn{8}{|c|}{ Panel D: nonlinearity Test } \\
\hline & $x^{2}$-stat. & d.f. & $P$ value & & & & \\
\hline DJI & 1035.713 & 5 & $<0.001$ & & & & \\
\hline STI & 300.714 & 5 & $<0.001$ & & & & \\
\hline
\end{tabular}

Note: Asterisks ${ }^{* * *},{ }^{* *},{ }^{*}$ indicate significance at the $1 \%, 5 \%, 10 \%$ level, respectively.

$t$-statistics are given in parentheses beneath the parameter estimates.

Panel A: $r_{t}=\varphi_{0}^{+}+\varphi_{1}^{+} r_{t-1}+\varphi_{2}^{+} m_{k, t}^{+}+\varepsilon_{t}^{+}$.

Panel B: $r_{t}=\varphi_{0}^{-}+\varphi_{1}^{-} r_{t-1}+\varphi_{2}^{-} m_{k, t}^{-}+\varepsilon_{t}^{-}$.

Panel C: $\varphi_{0}^{+}=\varphi_{0}^{-}=\varphi_{0} ; \varphi_{1}^{+}=\varphi_{1}^{-}=\varphi_{1} ; \varphi_{2}^{+}=\varphi_{2}^{-}=\varphi_{2}=0 ; \sigma_{\mu^{+}}^{2}=\sigma_{\mu^{-}}^{2}=\sigma_{\mu}^{2}\left(H_{0}^{3}\right)$.

nonlinearly depends on local high-low turning point (level and duration). Thus, the structure of trend part of the model is considered as Trend $(1 ; 1)$, not first-order autoregressive model $\operatorname{AR}(1)$.

In summary, the structure of the Trend-switching model for the indices is selected as Trend $(1 ; 1)-L(2,2 ; 2,2) D(1,1)$.

\subsection{Comparisons with Random Walk}

In this section, we will consider the forecast performance of Trend-LD model and compare our model with the traditional random walk model.

When the nonlinear model is used in predicting next day local trend $\left(\mu_{t+1}^{+}, \mu_{t+1}^{-}\right)$, it is necessary to know whether the direction of next day local trend is up or down, $I(k, t+1)$ is 1 or 0 . If the most recent turning point is high (low), next day local trend is down (up), $I_{t+1}=$ $1\left(I_{t+1}=0\right)$. In the definition, the high (low) turning point is the highest (lowest) points within the window of 20 days in radius. However, the most recent local maximum or minimum point is not necessarily a local high or low turning point, since number of the observations on the right of the most recent local maximum or minimum point is less than 20. So we decide whether to accept the most recent high (low) point based on the current available observation 
in two different cases. Denote the current observation by $Y_{t}$. Note that the data on the right side of $Y_{t}$ are unknown. The following gives two cases to decide whether a most recent local maximum or minimum point is a local turning point.

(1) If the most recent observation $Y_{t}$ is a local maxima (minima) in a given window $[t-w, t]$. From Definition 2.1, $Y_{t}$ is not seen as the local high (low) point in window $[t-w, t+w]$, since the observations between $(t, t+w]$ are yet unknown. In this case, we assume that the direction of the next observation $Y_{t+1}$ will continue to go up (down).

(2) If the current observation $Y_{t}$ is neither a local maxima nor minima in a given window $[t-w, t]$. Denote the window distance between the current observation $Y_{t}$ and the most recent local maxima or minima point (referred to as $M_{t}$ ) by $\tilde{w}$. The choice of a reference distance $c_{0}$ is important for our comparison. For the relative bias $Y_{t}$ and the window radius $w$ (20 days), we take $c_{0}=4$ days (one-fifth of the window radius $w$ ) as the referent distance. If $\tilde{w} \geq c_{0}$, then $M_{t}$ is regarded as a high or low turning point, and if $\tilde{w}<c_{0}$, we use the estimated local highlow level and up-down duration equation to predict the next local high turning point $\left(\widehat{h}, \widehat{d}^{+}\right)$or low turning point $\left(\widehat{l}, \widehat{d}^{-}\right)$, and then the direction of local trend is determined by comparing the most recent observed local maxima (minima) point with the predicted one. If the most recent observed local high (low) level is greater (less) than the predicted one and the most recent up (down) duration greater the predicted one, then $M_{t}$ is viewed as a high (low) turning point, and vice versa.

Using the above rules, we estimate the direction of the local trend. Denote the return of stock index by $R_{t+1}=\log \left(P_{t+1}\right)-\log \left(P_{t}\right)$ and the change of relative bias $Y_{t}$ by $r_{t+1}=Y_{t+1}-Y_{t}$. Then, from $Y_{t+1}=\log \left(P_{t+1}\right)-\mathrm{MA}\left(\log \left(P_{t}\right), 20\right)$, we have

$$
\begin{aligned}
r_{t+1} & =\left[\log \left(P_{t+1}\right)-\operatorname{MA}\left(\log \left(P_{t}\right), 20\right)\right]-\left[\log \left(P_{t}\right)-\operatorname{MA}\left(\log \left(P_{t-1}\right), 20\right)\right] \\
& =\left[\log \left(P_{t+1}\right)-\log \left(P_{t}\right)\right]-\left[\operatorname{MA}\left(\log \left(P_{t}\right), 20\right)-\operatorname{MA}\left(\log \left(P_{t-1}\right), 20\right)\right] \\
& =R_{t+1}-\operatorname{MA}\left(R_{t}, 20\right) .
\end{aligned}
$$

Rearrange the above formula, we derive the relationship between the return of stock index and the change of the relative bias as follows:

$$
R_{t+1}=r_{t+1}+\operatorname{MA}\left(R_{t}, 20\right) \text {. }
$$

Then, the one-step forecast of the return can be written as

$$
\widehat{R}_{t}(1)=\widehat{r}_{t}(1)+\operatorname{MA}\left(R_{t}, 20\right),
$$

where $\widehat{r}_{t}(1)$ is a predictor of $r_{t+1}$, and $\widehat{R}_{t}(1)$ is a predictor of $R_{t+1}$ in (5.2).

The forecast performance can be measured by comparing the real return with forecasted return on the direction and magnitude. It is well known that the daily return of stock index is very uncertain and difficult to predict. However, in practice, due to transaction costs, most investors do not trade frequently, but focus on the direction and magnitude of 
Table 6: $2 \times 2$ Contingent table.

\begin{tabular}{ccccc}
\hline & & & Forecast $\widehat{R}_{t}(1)$ & \\
& & Up & Down & Total \\
\hline \multirow{3}{*}{ Trend $\widetilde{R}_{t}(1)$} & Up & $M_{11}$ & $M_{12}$ & $M_{10}$ \\
& Down & $M_{21}$ & $M_{22}$ & $M_{20}$ \\
& Total & $M_{01}$ & $M_{02}$ & $M$ \\
\hline
\end{tabular}

local trend, with which our Trend-LD model is capable of dealing. However, the direction and magnitude of daily returns do not represent those of the local trend. In order to reduce the impact of random shocks on the local trend, we use the three-day moving average of the change of the bias (the real return can be seen as two parts: the unknown, deterministic expected local trend part and random shock part. As the stock prices can be affected by investors' complicated behavior biases, their behavior is difficult to be predicted, and the daily return does not reflect real trend. It is normal that the $R$-squared is very small in regression on short-term stock return, because the variation generated by random shock is responsible for most of the variation in stock return. When the time interval is smaller (daily), the effects of random shock on real return is greater, the accuracy of the prediction is clearly weaker, and the deterministic local trend part is relatively smaller or even submerged by the random shock. To reduce the effects of random shock, we use 3-day moving average of the real return as the proxy of the real local trend. Even the moving average is different from the local trend of real return, it is a better local trend proxy than real return, as the random shock is mostly averaged out. The purpose of our model is to predict the local trend of real return, not the real return that is easily affected by the random shock. The reason to use 3 -day is that if the longer the moving average is, the more it may lag behind the real local trend; if the shorter the moving average is, the weaker the random shock effects are removed), $\mathrm{MA}\left(r_{t+1}, 3\right)\left(=\left(r_{t+1}+r_{t}+\right.\right.$ $\left.\left.r_{t-1}\right) / 3\right)$, as a proxy of the local trend of the relative bias, that is, substitute $\mathrm{MA}\left(r_{t+1}, 3\right)$ into $r_{t+1}$ in formula (5.2), we have a proxy of the local trend of daily return, denoted by $\widetilde{R}_{t}(1)$, namely

$$
\tilde{R}_{t}(1)=\operatorname{MA}\left(r_{t+1}, 3\right)+\operatorname{MA}\left(R_{t}, 20\right) .
$$

The following two subsections are the forecasting performance comparisons of the direction and magnitude between Trend-LD model and random walk model.

Firstly, we consider the performance comparison of forecasting direction for our TrendLD model with random walk model. A common directional measure [49] is to use a $2 \times 2$ contingency table that summarizes the numbers of "rights" and "errors" of the model in predicting ups and downs of local trend of the return $R_{t+1}$ in the forecasting subsample, see Table 6. $M_{11}$ and $M_{22}$ indicate correct forecasts of the up and down direction, respectively. $M_{12}$ and $M_{21}$ indicate incorrect forecasts of the up and down direction, respectively. The Chi-squared statistic derived from the above contingent table is computed by

$$
x^{2}=\sum_{i=1}^{2} \sum_{j=1}^{2} \frac{\left(M_{i j}-M_{i 0} M_{0 j} / M\right)^{2}}{M_{i 0} M_{0 j} / M}
$$

which can be used to evaluate the performance of the model. A large $x^{2}$ signifies that the model outperforms the chance of random choice. Under some mild conditions, $x^{2}$ has an asymptotic chi-squared distribution with 1 degree of freedom. 
Table 7: $2 \times 2$ Contingent table.

\begin{tabular}{|c|c|c|c|c|c|c|c|c|c|c|}
\hline & & \multicolumn{3}{|c|}{$\begin{array}{c}\text { DJI } \\
\text { forecast } \widehat{R}_{t}(1)\end{array}$} & \multicolumn{3}{|c|}{$\begin{array}{l}\mathrm{SP} 500 \\
\text { forecast } \widehat{R}_{t}(1)\end{array}$} & \multicolumn{3}{|c|}{$\begin{array}{c}\text { FTSE } \\
\text { forecast } \widehat{R}_{t}(1)\end{array}$} \\
\hline & & Up & Down & Total & Up & Down & Total & Up & Down & Total \\
\hline \multirow{5}{*}{ Trend $\widetilde{R}_{t}(1)$} & Up & 1594 & 782 & 2376 & 1216 & 505 & 1721 & 473 & 225 & 698 \\
\hline & Down & 797 & 1103 & 1900 & 591 & 885 & 1472 & 245 & 350 & 595 \\
\hline & Total & 2391 & 1885 & 4276 & 1807 & 1386 & 3193 & 718 & 575 & 1293 \\
\hline & & \multicolumn{3}{|c|}{$\begin{array}{c}\text { STI } \\
\text { forecast } \widehat{R}_{t}(1)\end{array}$} & \multicolumn{3}{|c|}{$\begin{array}{c}\text { BSE } \\
\text { forecast } \widehat{R}_{t}(1)\end{array}$} & \multicolumn{3}{|c|}{$\begin{array}{l}\text { SZCI } \\
\text { forecast } \widehat{R}_{t}(1)\end{array}$} \\
\hline & & Up & Down & Total & Up & Down & Total & Up & Down & Total \\
\hline \multirow{3}{*}{ Trend $\widetilde{R}_{t}(1)$} & $\mathrm{Up}$ & 468 & 203 & 671 & 319 & 202 & 521 & 423 & 143 & 566 \\
\hline & Down & 237 & 307 & 544 & 130 & 292 & 422 & 223 & 219 & 442 \\
\hline & Total & 705 & 510 & 1215 & 449 & 494 & 943 & 646 & 362 & 1008 \\
\hline
\end{tabular}

Table 8: Forecast of the directions of trend and its test.

\begin{tabular}{lcccccc}
\hline & DJI & SP500 & FTSE & STI & BSE & SZCI \\
\hline$M_{11} / M_{10}$ & 0.671 & 0.707 & 0.678 & 0.698 & 0.612 & 0.747 \\
$M_{22} / M_{20}$ & 0.581 & 0.599 & 0.588 & 0.564 & 0.692 & 0.496 \\
$\left(M_{11}+M_{22}\right) / M$ & 0.631 & 0.657 & 0.637 & 0.638 & 0.648 & 0.637 \\
$X^{2}$ & 270.697 & 300.586 & 91.954 & 84.546 & 86.514 & 63.584 \\
$P$ value & $<0.001$ & $<0.001$ & $<0.001$ & $<0.001$ & $<0.001$ & $<0.001$ \\
\hline
\end{tabular}

Table 7 shows results of the numbers of "rights" and "errors" of the model in 1-step predicting ups and downs of local trend of the return in the six forecasting subsamples. Table 8 summarizes the right and error rates of directional prediction and its tests. the DJI has the smallest right rate, $63.1 \%$ and the SP500 has the largest, $65.7 \%$. The $P$ values of statistic $x^{2}$ are almost zero. This shows that the dynamic behavior of Trend-LD model outperforms significantly the random walk model for all the six stock indices. In addition, the right rates of up direction $\left(M_{11} / M_{10}\right)$ are greater than those of down direction $\left(M_{22} / M_{20}\right)$ except the BSE. Indeed, this can be observed intuitively from Figure 1. In this figure, we find that, when the price rises, the local trend of index is more regular and easily predicted than the case when the price falls. This may result from the complicated, asymmetric psychology factors of most of investors, such as diminishing marginal utility, greed, and fear. Moreover, interestingly, the best and worst rate of forecast of up direction is $61.2 \%$ of the BSE and $74.5 \%$ of the SZCI, respectively. For the down direction, conversely, the right rate of the SZCI is $49.6 \%$ and the BSE is $69.2 \%$, respectively. That is, the best and worst rate of forecast of the up-down direction always appears in the market of underdeveloped countries.

Secondly, we consider the comparison of forecasting magnitude for the two models above. Assume that the predicting error $e_{1 t}$ of the Trend-LD model is the difference between forecast return $\widehat{R}_{t}(1)$ and the proxy of local trend $\widetilde{R}_{t}(1)$, and the predicting error $e_{2 t}$ of random walk hypothesis is the difference between 0 and $\widetilde{R}_{t}(1)$. Denote $d_{t}=e_{1 t}^{2}-e_{2 t}^{2}$, the forecast performance of the magnitude of local trend is tested by a DM statistic [50]

$$
\mathrm{DM}=\frac{\bar{d}}{\sqrt{2 \pi \hat{f}_{d}(0) / T}},
$$


Table 9: The statistic DM and its test with window radius $w=20$.

\begin{tabular}{lcccccc}
\hline & DJI & SP500 & FTSE & STI & BSE & SZCI \\
\hline DM & -4.524 & -4.612 & -2.207 & -2.793 & -2.659 & -3.060 \\
$P$ value & $<0.001$ & $<0.001$ & 0.014 & 0.003 & 0.004 & 0.001 \\
\hline
\end{tabular}

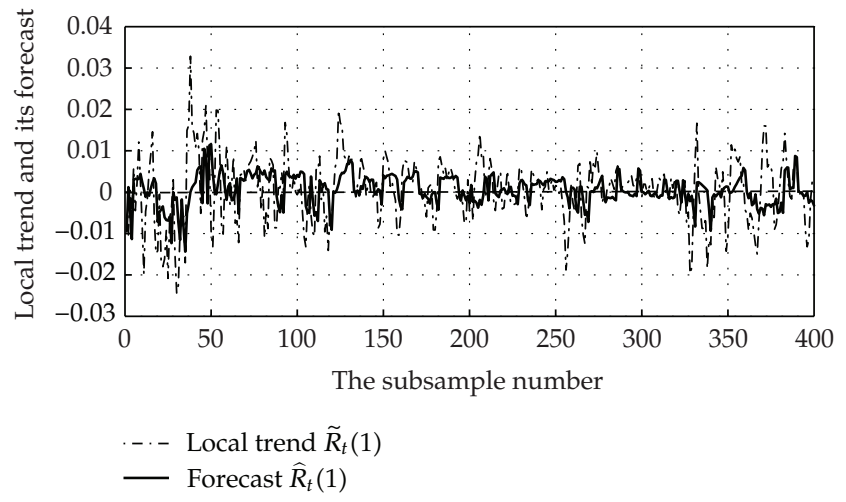

Figure 3: The local trend of the returns of the DJI and its forecasts (2010.03.29-2010.08.18).

where $\bar{d}$ is the sample mean of the loss differential series $d_{1}, d_{2}, \ldots, d_{T}, \widehat{f}_{d}(0)$ is a consistent estimate of $f_{d}(0), f_{d}(\cdot)$ is the spectral density of series $\left\{d_{t}\right\}$. Under the null hypothesis of equal predictive accuracy, the DM statistic is asymptotically standard normal distribution $N(0,1)$. In Table 9, the $P$ values of the DM statistic show that the magnitude forecasts of local trend model all significantly outperform the random walk model.

In Figure 3 (as there are 4276 forecasted daily returns, the figure will be too congested to convey any details if we plot the total time series. As a result, we only show part of the forecasted results), the dash-dot line is the proxy of the real local trend $\widetilde{R}_{t}(1)$, the solid line is the forecast of the real local trend $\widehat{R}_{t}(1)$. Even if the forecast of the direction and magnitude of local trend have some errors, the overall forecast is good. Furthermore, the direction and magnitude of the local trend and its prediction show inertia (duration), which is different from the dynamic of the mean-reversion model and the error correction model that the direction and magnitude of the local trend will immediately change and it will go back to the long-term mean level or equilibrium when the process deviates from a long-term mean level or equilibrium.

To summarize, the proposed Trend-LD model can efficiently reflect the fluctuation characteristics of financial time series, such as the inertia or the duration of the local updown trend, the asymmetric dependency of the alternate local high-low level, the level and duration clustering and has many properties that beyond the traditional random walk model.

\section{Conclusions}

Trend-LD model, in essence, is a conditional regime-switching (or time-varying twodimensional thresholds) model, its threshold (local high-low levels and up-down durations or the high-low turning points) is not fixed, but conditionally dependent on the past ones. This means that the proposed model can incorporate longer-term, salient history information 
into trend-switching. Therefore, it is more flexible than the traditional random walk, meanreversion, TAR, STAR and MSAR models.

Our model may introduce to some more interesting further studies: (1) The choices of the order of moving average $m$ and the window radius $w$, and the determination of the local trend change is a challenge and interesting problem for further exploring. (2) Comparisons of our model with mean reversion, TAR, STAR, and MSAR models for other time series data are also meaningful.

\section{Acknowledgments}

This work is supported by National Natural Science Foundations of China (no. 71001045, 10971162), Natural Science Foundation of Jiangxi Province of China (no. 20114BAB211008), and Jiangxi University of Finance and Economics Support Program Funds for Outstanding Youths. The authors thank the Editor and referees for their helpful comments.

\section{References}

[1] B. Robert, "A brief account of microscopical observations made in the months of June, July and August (1827), on the particles contained in the pollen of plants; and on the general existence of active molecules in organic and inorganic bodies," Philosophical Magazine, vol. 4, pp. 161-173, 1828.

[2] K. Pearson, "The problem of the random walk," Nature, vol. 72, p. 294, 1905.

[3] G. E. Uhlenbeck and L. S. Ornstein, "On the theory of Brownian motion," Physical Review, vol. 36, 1930.

[4] A. K. Dixit and R. S. Pindyck, Investment under Uncertainty, Princeton University Press, Princeton, NJ, USA, 1994.

[5] G. E. P. Box and G. M. Jenkins, Times Series Analysis. Forecasting and Control, Holden-Day, San Francisco, Calif, USA, 1970.

[6] R. F. Engle and C. W. J. Granger, “Co-integration and error correction: representation, estimation, and testing," Econometrica, vol. 55, no. 2, pp. 251-276, 1987.

[7] H. Tong and K. S. Lim, "Threshold autoregression, limit cycles and cyclical data," Journal of the Royal Statistical Society, Series B, vol. 42, pp. 245-292, 1980.

[8] H. Tong, Non-Linear Time Series: A Dynamical Systems Approach, Oxford University Press, Oxford, UK, 1990.

[9] T. Teräsvirta, "Specification, estimation, and evaluation of smooth transition autoregressivemodels," Journal of the American Statistical Association, vol. 89, pp. 208-218, 1994.

[10] T. Teräsvirta, "Modelling economic relationships with smooth transition regressions," in Handbook of Applied Economic Statistics, A. Ullah and D. E. A. Giles, Eds., pp. 507-552, Marcel Dekker, New York, NY, USA, 1998.

[11] J. D. Hamilton, "A new approach to the economic analysis of nonstationary time series and the business cycle," Econometrica, vol. 57, no. 2, pp. 357-384, 1989.

[12] J. D. Hamilton, "Specification testing in Markov-switching time-series models," Journal of Econometrics, vol. 70, no. 1, pp. 127-157, 1996.

[13] C.-J. Kim, "Dynamic linear models with Markov-switching," Journal of Econometrics, vol. 60, no. 1-2, pp. 1-22, 1994.

[14] J. Cai, "A Markov model of switching-regime ARCH," Journal of Business \& Economic Statistics, vol. 12, pp. 309-316, 1994.

[15] A. Ang and G. Bekaert, "International asset allocation with regime shifts," Review of Financial Studies, vol. 15, pp. 1137-1187, 2002.

[16] A. Ang and G. Bekaert, "Regime switches in interest rates," Journal of Business E Economic Statistics, vol. 20, no. 2, pp. 163-182, 2002.

[17] L. Calvet and A. Fisher, "How to forecast long-run volatility: regime-switching and the estimation of multifractal processes," Journal of Financial Econometrics, vol. 2, pp. 49-83, 2004.

[18] T. Davig, "Regime-switching debt and taxation," Journal of Monetary Economics, vol. 51, pp. 837-859, 2004. 
[19] S. Beckers, "Variances of security price returns based on high, low, and closing prices," Journal of Business, vol. 56, pp. 97-112, 1983.

[20] S. Alizadeh, M. W. Brandt, and F. X. Diebold, "Range-based estimation of stochastic volatility models," Journal of Finance, vol. 57, pp. 1047-1091, 2002.

[21] R. Y. Chou, "Forecasting financial volatilities with extreme values: the conditional autoregressive range (CARR) model," Journal of Money, Credit and Banking, vol. 37, pp. 561-582, 2005.

[22] R. Y. Chou, "Modeling the asymmetry of stock movements using price ranges," Advances in Econometrics, vol. 20, pp. 231-257, 2006.

[23] Y.-L. Cheung, Y.-W. Cheung, and A. T. K. Wan, "A high-low model of daily stock price ranges," Journal of Forecasting, vol. 28, no. 2, pp. 103-119, 2009.

[24] R. F. Engle and J. R. Russell, “Autoregressive conditional duration: a new model for irregularly spaced transaction data," Econometrica, vol. 66, no. 5, pp. 1127-1162, 1998.

[25] L. Bauwens and P. Giot, "The logarithmic ACD model: an application to the bid-ask quote process of three NYSE stocks," Annals of Economics and Statistics, vol. 60, pp. 117-149, 2000.

[26] M. Y. Zhang, J. R. Russell, and R. S. Tsay, "A nonlinear autoregressive conditional duration model with applications to financial transaction data," Journal of Econometrics, vol. 104, no. 1, pp. 179-207, 2001.

[27] L. Bauwens and D. Veredas, "The stochastic conditional duration model: a latent variable model for the analysis of financial durations," Journal of Econometrics, vol. 119, no. 2, pp. 381-412, 2004.

[28] R. F. Engle and J. R. Russell, "Forecasting the frequency of changes in quoted foreign exchange prices with autoregressive conditional duration model," Journal of Empirical Finance, vol. 4, pp. 187-212, 1997.

[29] J. Jasiak, "Persistence in intertrade durations," Finance, vol. 19, pp. 166-195, 1998.

[30] R. F. Engle, "The econometrics of ultra-high frequency data," Econometrica, vol. 68, pp. 1-22, 2000.

[31] J. Grammig and K.-O. Maurer, "Non-monotonic hazard functions autoregressive conditional duration model," Econometrics Journal, vol. 3, pp. 16-38, 2000.

[32] M. Y. Zhang, J. R. Russell, and R. S. Tsay, "A nonlinear autoregressive conditional duration model with applications to financial transaction data," Journal of Econometrics, vol. 104, no. 1, pp. 179-207, 2001.

[33] J. Grammig and M. Wellner, "Modeling the interdependence of volatility and inter-transaction duration processes," Journal of Econometrics, vol. 106, no. 2, pp. 369-400, 2002.

[34] M. E. Holder, M. Qi, and A. K. Sinha, "The impact of time duration between trades on the price of treasury note futures contracts," Journal of Futures Markets, vol. 24, pp. 965-980, 2004.

[35] S. X. Lin and M. N. Tamvakis, "Effects of NYMEX trading on IPE brent crude futures markets: a duration analysis," Energy Policy, vol. 32, pp. 77-82, 2004.

[36] S. M. Focardi and F. J. Fabozzi, "An autoregressive conditional duration model of credit-risk contagion," Journal of Risk Finance, vol. 6, pp. 208-225, 2005.

[37] S. Manganelli, "Duration, volume and volatility impact of trades," Journal of Financial Markets, vol. 8, pp. 377-399, 2005.

[38] L. Bauwens, "Econometric analysis of intra-daily activity on Tokyo stock exchange," Forthcoming in Monetary and Economic Studies, vol. 24, 2006.

[39] A. F. Burns and W. C. Mitchell, Measuring Business Cycles, New York, NY, USA, 1946.

[40] M. Z. Kontolemis and D. Osborn, "Business cycles for G7 and European countries," Journal of Business, vol. 70, pp. 249-279, 19972.

[41] F. Canova, “Detrending and business cycle facts," Journal of Monetary Economics, vol. 41, pp. 475-512, 1998.

[42] F. Canova, "Detrending and business cycles facts: a user's guide," Journal of Monetary Economics, vol. 41, pp. 522-540, 1998.

[43] D. H. Hathaway, "The solar cycle," Living Reviews in Solar Physics, vol. 7, p. 1, 2010.

[44] W. E. Wecker, "Predicting the turning points of a time series," The Journal of Business, vol. 52, no. 1, pp. 35-50, 1979.

[45] M. Amstad, "On the use of Markov switching models applied to business survey data for the prediction of turning points," in Proceedings of the 24th CIRET Conference, K. H. Oppenlaender, Ed., pp. 3-26, Wellington, New Zealand, 2000.

[46] D. Kugiumtzis, "Local prediction of turning points of oscillating time series," Physical Review E, vol. 78, no. 3, pp. 1-7, 2008.

[47] J. M. Durland and T. H. McCurdy, "Duration-dependent transitions in a Markov model of U.S. GNP growth," Journal of Business \& Economic Statistics, vol. 12, no. 3, pp. 279-288, 1994.

[48] J. D. Hamilton, Time Series Analysis, Princeton University Press, Princeton, NJ, USA, 1994. 
[49] R. S. Tsay, Analysis of Financial Time Series, John Wiley \& Sons Inc., Hoboken, NJ, USA, Third edition, 2010.

[50] F. X. Diebold and R. S. Mariano, "Comparing predictive accuracy," Journal of Business and Economic Statistics, vol. 13, pp. 253-263, 1995. 


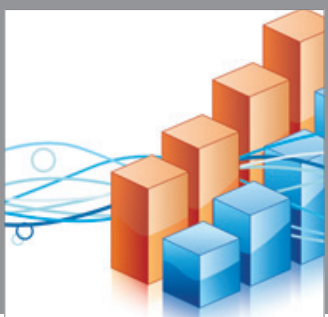

Advances in

Operations Research

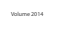

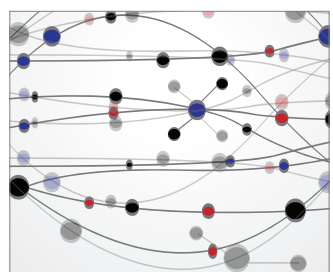

\section{The Scientific} World Journal
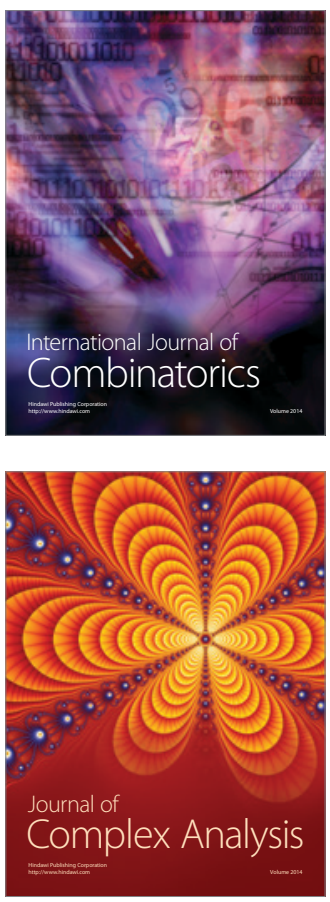

International Journal of

Mathematics and

Mathematical

Sciences
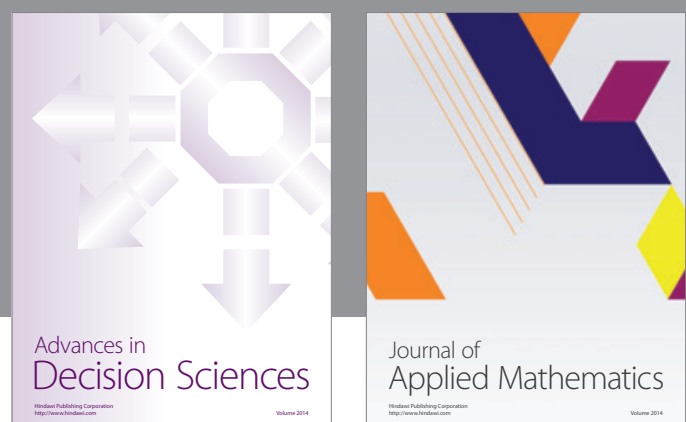

Journal of

Applied Mathematics
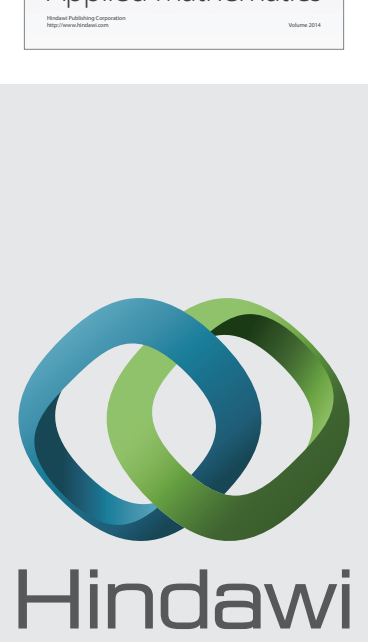

Submit your manuscripts at http://www.hindawi.com
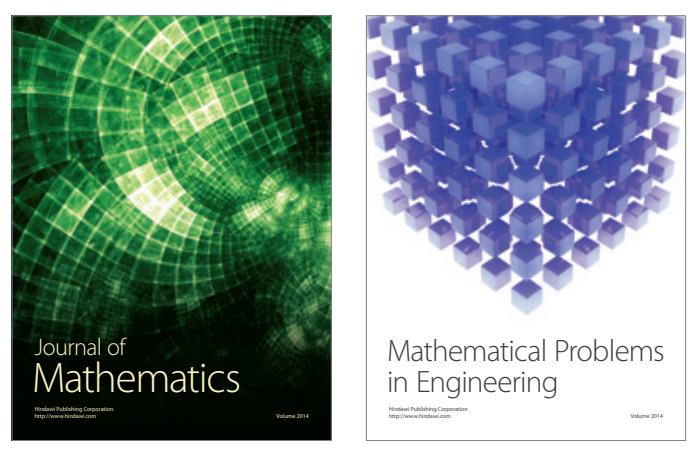

Mathematical Problems in Engineering
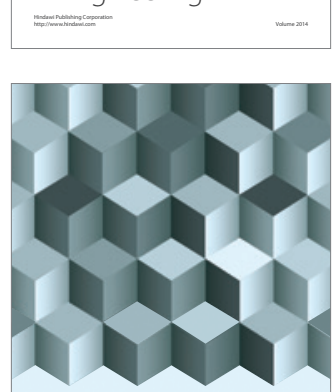

Journal of

Function Spaces
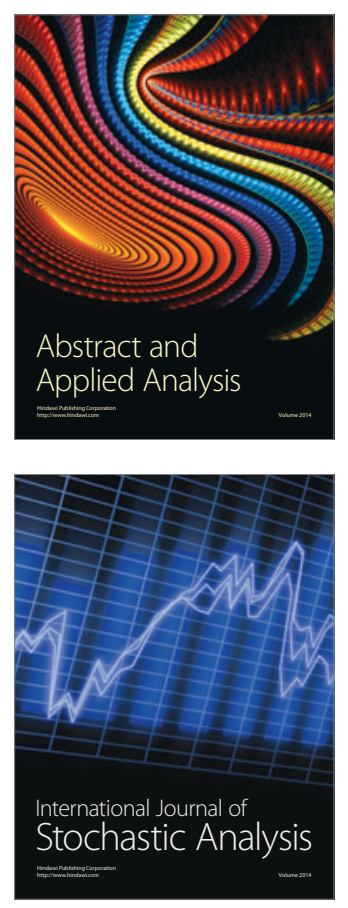

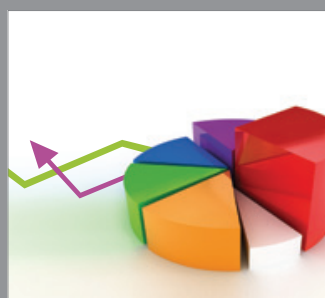

ournal of

Probability and Statistics

Promensencen
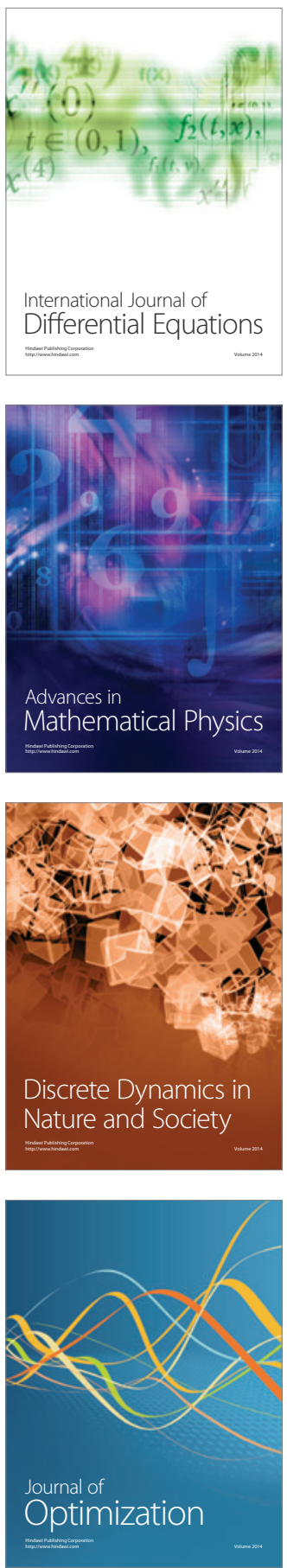